\title{
Relatório de pareceristas da Revista de Sociologia e Política em 2013
}

Board of Reviewers of Revista de Sociologia e Política in 2013

Relación de evaluadores de la Revista de Sociologia e Política en 2013

Adrian Gurza Lavalle (USP) ad hoc

Adriano Luís Duarte (UFSC) ad hoc

Adriano Nervo Codato (UFPR)

Afonso Albuquerque (UFF) ad hoc

Alberto Montoya Correa Palacios Junior (ESPM) ad hoc

Alexandre Gori Maia (UNICAMP) ad hoc

Alexsandro Eugênio Pereira (UFPR)

Ana Cláudia Niedhardt Capella (Unesp) ad hoc

Ana Zandwais (UFRGS) ad hoc

André Moreira Cunha (UFRGS) ad hoc

André Silva (USP) ad hoc

Andréa Benetti Carvalho de Oliveira (UFPR) ad hoc

Andréa Carolina Schvartz Peres (CEBRAP) ad hoc

Andrea Fachel Leal (UFRGS) ad hoc

Andréa Maria Silveira (UFMG) ad hoc

Ângela Maria de Randolpho Paiva (PUC-RJ) ad hoc

Angelo Brás Fernandes Callou (UFRPE) ad hoc

Aníbal Jáuregui (UBA, Argentina)

Anita Helena Schlesener (UTP) ad hoc

Antônio Carlos Alkmim dos Reis (IBGE) ad hoc

Antônio Carlos Lessa (UnB) ad hoc

Armando Boito Jr (UNICAMP) ad hoc

Arnaldo Mazzei Nogueira (USP) ad hoc

Bárbara Gomes Lamas (UERJ) ad hoc

Bruna Gisi Martins de Almeida (USP) ad hoc

Camilo Aggio (UFBA) ad hoc

Carlos Acevedo Rodríguez (Universidad Arturo Prat, Chile) ad hoc

Carlos Alberto Rocha (PUC-MG) ad hoc

Carolina Biernat (Universidad Nacional de Quilmes, Argentina) ad hoc

Cecília Olivieri (USP) ad hoc

César Augusto Da Ros (UFRRJ) ad hoc

Charles Wood (University of Florida, USA) ad hoc

Claudia Feres Faria (UFMG) ad hoc

Claudio Fabián Belini (Universidad de Buenos Aires, Argentina) ad hoc

Cláudio Gonçalves Couto (FGV-SP) ad hoc

Cláudio Júnior Damin (UFRGS) ad hoc

Cleber da Silva Lopes (UEL) ad hoc

Corival Carmo (UFS) ad hoc

Cristiane Brum Bernardes (CEFOR/Câmara dos Deputados) ad hoc

Cristiane Correa Batista Santos (UNIRIO) ad hoc

Cristiane Lucena Carneiro (USP) ad hoc

Cristiano Ferri Soares de Faria (CEFOR/Câmara dos Deputados) ad hoc

Cristina Soreanu Pecequilo (UNIFESP) ad hoc

Débora Cristina Rezende de Almeida (UnB) ad hoc

Débora Messenberg (UnB) ad hoc

Debora Regina Pastana (UFU) ad hoc

Denilson Bandeira Coêlho (UnB) ad hoc 
Denise Barbosa Gros (FEE) ad hoc

Denise Cristina Vitale Ramos Mendes (UFBA) ad hoc

Denise Paiva (UFG) ad hoc

Diego Pautasso (ESPM) ad hoc

Diego Santos Vieira de Jesus (PUC-RJ) ad hoc

Edemar Rotta (UFFS) ad hoc

Edileusa Godói de Sousa (UFU) ad hoc

Ednaldo Aparecido Ribeiro (UFPR) ad hoc

Eduardo Cavieres Fernández (Universidad de Playa Ancha, Chile) ad hoc

Elaine Regina Aguiar Amorim (UNICAMP) ad hoc

Eleonora Schettini Martins Cunha (UFMG) ad hoc

Emerson Giumbelli (UFRGS) ad hoc

Emerson Urizzi Cervi (UFPR)

Emil Sobottka (PUC-RS) ad hoc

Everton Rodrigo Santos (FEEVALE) ad hoc

Fabiana Augusta Alves Jardim (USP) ad hoc

Fabiana Luci de Oliveira (UFSCar) ad hoc

Fábio Fonseca Figueiredo (UFRN) ad hoc

Fabrício Mazocco (UFSCar) ad hoc

Fabricio Ricardo de Limas Tomio (UFPR)

Feliciano de Sá Guimarães (ESPM) ad hoc

Félix Aguirre (Universidad de Valparaíso, Chile) ad hoc

Fernanda Mello Sant'Anna (USP) ad hoc

Fernando Bessa Ribeiro (Universidade do Minho, Portugal) ad hoc

Fernando Coelho (USP) ad hoc

Fernando de Barros Filgueiras (UFMG) ad hoc

Fernando Ferrari Filho (UFRGS) ad hoc

Fernando Manuel Rocha da Cruz (UFRN) ad hoc

Flávia Biroli (UnB) ad hoc

Flávio da Cunha Rezende (UFPE) ad hoc

Francisco Paulo Jamil Almeida Marques (UFC) ad hoc

Gabriel Avila Casalecchi (UFMG)

Gabriel Stecher (Universidad Nacional del Comahue - Argentina) ad hoc

Gabriela Tarouco (UFPE) ad hoc

Geraldo Magella Neres (UNIOESTE) ad hoc

Giane Silvestre (UFSCar) ad hoc

Giovanni Semeraro (UFF) ad hoc

Gonzalo Assusa (Universidad Nacional de Córdoba, Argentina) ad hoc

Guilherme de Azevedo Dantas (UFRJ) ad hoc

Guilherme G. de F. Xavier Sobrinho (FEE) ad hoc

Guilherme Reis Pereira (UFRN) ad hoc

Gustavo Biscaia de Lacerda (UFPR)

Gustavo Melo Silva (UFSJ) ad hoc

Hélder Ferreira do Vale (European University Institute - Florença, Itália) ad hoc

Helder Gordim da Silveira (PUC-RS) ad hoc

Helena Margarido Moreira (USP) ad hoc

Hélio Ricardo do Couto Alves (FURG) ad hoc

Heloisa Dias Bezerra (UFG) ad hoc

Hemerson Luiz Pase (UFPel) ad hoc

Ignacio José Godinho Delgado (UFJF) ad hoc

Igor Ferraz da Fonseca (UnB) ad hoc

Ivanete Boschetti (UnB) ad hoc

Ivann Carlos Lago (UFFS) ad hoc

Janina Onuki (USP) ad hoc

Javier Amadeo (UNIFESP) ad hoc 
Javier Vadell (PUC-MG) ad hoc

Jerônimo Oliveira Muniz (UFMG) ad hoc

Joana Tereza Vaz de Moura (UFRN) ad hoc

John Cunha Comerford (UFRJ) ad hoc

José Alberto Gomes Precioso (Universidade do Minho, Portugal) ad hoc

José Antônio da Silva Júnior (UNESP/Araraquara) ad hoc

José Luís Sanfelice (UNICAMP) ad hoc

José Maurício Domingues (UERJ) ad hoc

Josep Vidal (UFPA) ad hoc

Julia Moretto Amâncio (UFLA) ad hoc

Julian Borba (UFSC) ad hoc

Kelly Cristina Costa Soares (UFCG) ad hoc

Léa Guimarães Souki (PUC-MG) ad hoc

Leany Barreiro Lemos (UnB) ad hoc

Leo Peixoto Rodrigues (UFPEL) ad hoc

Leonardo Biscaia de Lacerda (UFPR) ad hoc

Leonardo Ramos (PUC-Minas) ad hoc

Livia de Tommasi (UFF) ad hoc

Lorena Madruga Monteiro (UFRGS) ad hoc

Lucas Massimo T A de Souza (UFPR)

Luciana Aliaga (UEL) ad hoc

Luciana Dias de Lima (FIOCRUZ) ad hoc

Luciana Fernandes Veiga (UFPR)

Luciana Leite Lima (UFRGS) ad hoc

Lucilia de Almeida Neves Delgado (UnB) ad hoc

Luisina Perelmiter (Universidad Nacional de San Martín, Argentina) ad hoc

Luiz Eduardo Motta (UFRJ) ad hoc

Luiz Paulo Rouanet (UFSJR) ad hoc

Malena Rabhein Rodrigues (CEFOR/Câmara dos Deputados) ad hoc

Marçal de Menezes Paredes (PUC-RS) ad hoc

Marcel Bursztyn (UnB) ad hoc

Marcello Cavalcanti Barra (MPOG) ad hoc

Marcelo Carvalho Rosa (UnB) ad hoc

Marcelo da Silveira Campos (USP) ad hoc

Marco Antônio Carvalho Teixeira (FGV) ad hoc

Marcos César Alvarez (USP) ad hoc

Marcos Del Roio (UNESP) ad hoc

Marcos Novaro (Cipol, UBA, Argentina) ad hoc

María Angélica Cruz Contreras (Universidad de Valparaíso, Chile) ad hoc

Maria Angélica Oliva Ureta (Universidad de Talca, Chile) ad hoc

Maria Carla Corrochano (UFSCar) ad hoc

María Gabriela Brandán Zehnder (Universidad Católica de Córdoba, Argentina) ad hoc

Maria Luiza Lins e Silva Pires (UFRPE) ad hoc

Maria Odete Alves (UnB) ad hoc

Mariana Gené (UBA-Conicet, Argentina) ad hoc

Marta Arretche (USP) ad hoc

Marta Fernández Moreno (PUC-RJ) ad hoc

Matheus de Carvalho Hernandez (UFGD) ad hoc

Maurício Michel Rebello (UFRGS) ad hoc

Mehmet Çete (Erciyes University, Kayseri, Turkey) ad hoc

Michele Polline Veríssimo (UFU) ad hoc

Miguel Gualano Godoy (UFPR) ad hoc

Monica Schiavinatto (UnB) ad hoc

Nildo Avelino (UFPB) ad hoc

Octavio Augusto Camargo Conceição (UFRGS) ad hoc 
Odaci Luiz Coradini (UFRGS) ad hoc

Oliveiros S. Ferreira (USP) ad hoc

Oswaldo Truzzi (UFSCar) ad hoc

Paolo Ricci (USP) ad hoc

Patricia Berrotarán (Universidad Nacional de Quilmes, Argentina) ad hoc

Paula Regina Pereira Marcelino (USP) ad hoc

Paulo Peres (UFRGS) ad hoc

Paulo Roberto de Almeida (Instituto Rio Branco) ad hoc

Pedro Floriano Ribeiro (UFSCar) ad hoc

Pedro Luiz Costa Cavalcante (MPOG) ad hoc

Pedro Paulo Zahluth Bastos (UNICAMP) ad hoc

Pedro Robson Pereira Neiva (CEBRAP) ad hoc

Pedro Simões (UFRJ) ad hoc

Petronio De Tilio Neto (MACKENZIE) ad hoc

Rachel Callai Bragatto (UFPR) ad hoc

Rafael Cardoso Sampaio (UFBA) ad hoc

Rafael da Silva (UEM) ad hoc

Rafael Nunes Magalhães (USP) ad hoc

Ramon Blaco (Universidade de Coimbra, Portugal) ad hoc

Raphael Padula (UFRJ) ad hoc

Raúl Bernal-Meza (Universidad Nacional del Centro, Argentina) ad hoc

Renan Springer de Freitas (UFMG) ad hoc

Robert Bonifácio da Silva (UFMG) ad hoc

Robson Coelho Cardoch Valdez (UFRGS) ad hoc

Rodrigo Rossi Horochovski (UFPR) ad hoc

Rogério Medeiros (UFPB) ad hoc

Ruth Felder (UBA, Argentina) ad hoc

Salim Cevik (Bilkent University, Turkey) ad hoc

Samira Kauchakje (PUC-PR) ad hoc

Sandra Regina de Abreu Pires (UEL) ad hoc

Sebastián Valverde (FFyL-UBA/CONICET, Argentina) ad hoc

Sergio Barreira de Faria Tavolaro (UnB) ad hoc

Sérgio Soares Braga (UFPR)

Susana Ethel Roitman (Universidade Nacional del Villa María, Argentina) ad hoc

Tatiana Berringer (UNICAMP) ad hoc

Thaís Battibugli (UNESP/Marília) ad hoc

Thais Silva Menezes (USP) ad hoc

Tiago Bahia Losso (UFSC) ad hoc

Umberto Guarnier Mignozzetti (USP) ad hoc

Vânia Cardoso da Motta (UERJ) ad hoc

Vera Alves Cepêda (UFSCAR) ad hoc

Viviani Corrêa Teixeira (UFSC) ad hoc

Vladimyr Lombardo Jorge (UFRRJ) ad hoc

Wilson da Silva Gomes (UFBA) ad hoc

Wilson José Ferreira de Oliveira (Ufpel) ad hoc 